\title{
Exposure of mouse embryonic pancreas to metformin enhances the number of pancreatic progenitors
}

\author{
Brigid Gregg • Lynda Elghazi • Emilyn U. Alejandro • Michelle R. Smith • \\ Manuel Blandino-Rosano • Deena El-Gabri • Corentin Cras-Méneur • \\ Ernesto Bernal-Mizrachi
}

Received: 23 July 2014 / Accepted: 28 August 2014 / Published online: 24 September 2014

(C) Springer-Verlag Berlin Heidelberg 2014

\begin{abstract}
Aims/hypothesis Developing beta cells are vulnerable to nutrient environmental signals. Early developmental processes that alter the number of pancreatic progenitors can determine the number of beta cells present at birth. Metformin, the most widely used oral agent for treating diabetes, alters intracellular energy status in part by increasing AMP-activated protein kinase (AMPK) signalling. This study examined the effect of metformin on developing pancreas and beta cells.

Methods Pancreatic rudiments from CD-1 mice at embryonic day 13.0 (E13.0) were cultured with metformin, 5-aminoimidazole-4-carboxamide-1- $\beta$-D-ribofuranoside (AICAR, an AMPK activator) or vehicle control in vitro. In another set of studies, pregnant C57BL/6 mice were treated with metformin throughout gestation. Embryonic (E14.0) and neonatal pancreases were then analysed for their morphometry.

Results In vitro metformin treatment led to an increase in the proliferation and number of pancreatic duodenal homeobox 1-positive $\left(\mathrm{PDX}^{+}{ }^{+}\right)$progenitors. These results were reproduced by in vitro culture of embryonic pancreas rudiments with AICAR, suggesting that AMPK activation was involved. Similarly, metformin administration to pregnant dams induced
\end{abstract}

Brigid Gregg and Lynda Elghazi contributed equally to this work.

B. Gregg $\cdot$ M. R. Smith $\cdot$ D. El-Gabri

Department of Pediatrics, Division of Endocrinology, Diabetes and

Metabolism, University of Michigan, Ann Arbor, MI, USA

L. Elghazi • E. U. Alejandro • M. R. Smith • M. Blandino-Rosano •

C. Cras-Méneur $\cdot$ E. Bernal-Mizrachi $(\bowtie)$

Department of Internal Medicine, Division of Metabolism,

Endocrinology and Diabetes, Brehm Center for Diabetes Research,

University of Michigan, Ann Arbor, MI 48109-0678, USA

e-mail: ebernal@umich.edu

E. Bernal-Mizrachi

VA Ann Arbor Healthcare System, Ann Arbor, MI, USA an increase in both $\mathrm{PDX}_{1}^{+}$and neurogenin 3-positive progenitors in the embryonic pancreas at E14.0 and these changes resulted in an increased beta cell fraction in neonates.

Conclusions/interpretation These results indicate that exposure to metformin during gestation modulates the early steps of beta cell development (prior to E14.0) towards an increase in the number of pancreatic and endocrine progenitors. These changes ultimately result in a higher beta cell fraction at birth. These findings are of clinical importance given that metformin is currently used for the treatment of gestational diabetes.

Keywords AICAR - AMPK - Developmental programming $\cdot$ Metformin $\cdot$ mTOR $\cdot$ Pancreas development

\begin{tabular}{ll}
\multicolumn{2}{l}{ Abbreviations } \\
ACC & Acetyl-CoA carboxylase \\
AICAR & 5-Aminoimidazole-4-carboxamide- \\
& 1- $\beta$-D-ribofuranoside \\
AMPK & AMP-activated protein kinase \\
mTOR & Mammalian target of rapamycin \\
mTORC1 & mTOR complex 1 \\
NGN3 & Neurogenin 3 \\
PDX1 & Pancreatic duodenal homeobox 1
\end{tabular}

Introduction

Type 2 diabetes is one of the most prevalent conditions affecting human health today. It is understood that both genetic and environmental factors contribute to type 2 diabetes risk [1], and one important environmental factor is maternal nutrition during pregnancy [2]. Developing beta cells have been shown to be critically sensitive to nutrient status [3-6]. Experimental models of metabolic stress during pancreatic development 
show permanent impairments in offspring beta cell mass and function [7-10]. This phenomenon, termed beta cell programming, is also seen in observational studies in humans [11].

Pancreas development begins at embryonic day (E)8.5 within a region of the endoderm [12]. Pancreatic duodenal homeobox 1-positive $\left(\mathrm{PDX}^{+}\right)$cells represent a population of progenitor cells for all mature pancreatic cells [13]. These undifferentiated precursor cells can be specified towards the endocrine lineage by the expression of neurogenin 3 (NGN3) [14]. After the expression of a cascade of transcription factors, these cells differentiate into the five endocrine cell types: alpha cells (glucagon), beta cells (insulin), delta cells (somatostatin), PP cells (pancreatic polypeptide) and epsilon cells (ghrelin) (reviewed in $[13,15])$.

The mechanisms by which nutrition-related changes influence beta cell development are unclear, but signalling pathways that respond to changes in energy status are prime candidates. Mammalian target of rapamycin (mTOR) is a nutrient sensor that has been shown to be important for beta cell mass and function in rodent models $[16,17]$. The role of mTOR complex 1 (mTORC1) signalling in the regulation of mature beta cell mass and proliferation has been established $[18,19]$. However, an understanding of the role of this pathway in the developing beta cell is only indirect [20]. Metformin, the most widely used oral glucose-lowering agent, has been demonstrated to decrease mTORC1 activity through various mechanisms including AMP-activated kinase (AMPK) induction [21]. Metformin also acts on nutrient signalling pathways via AMPK-independent mechanisms $[22,23]$. Metformin is being studied for use during pregnancy in polycystic ovary syndrome and gestational diabetes [24, 25]. However, the implications for alterations in pancreatic embryonic development induced by metformin have not been characterised. We sought to directly examine the impact of metformin on pancreatic development using both an in vitro and an in vivo approach to assess the resultant alterations in the embryonic and neonatal pancreas.

\section{Methods}

Pancreatic bud culture in vitro Pancreatic rudiments were dissected from E13.0 embryos (the morning of vaginal plug was E0.5) of CD-1 dams purchased from Charles River (Wilmington, MA, USA) according to the University of Michigan School of Medicine-approved protocols. Pancreatic rudiments were cultured, as described previously [26, 27], for $72 \mathrm{~h}$ in DMSO with or without $2 \mathrm{mmol} / \mathrm{l}$ metformin or $1 \mathrm{mmol} / 1$ 5-aminoimidazole-4-carboxamide-1- $\beta$-Dribofuranoside (AICAR) (Sigma-Aldrich, St Louis, MO, USA). After culture, embryonic rudiments were fixed in $3.7 \%$ formalin in PBS and pre-embedded in Histogel
(Thermo Scientific, Kalamazoo, MI, USA) for paraffin embedding.

In vivo metformin programming mouse model Eight-weekold virgin C57Bl6 mice were purchased from Jackson Laboratories (Bar Harbor, ME, USA) and adapted to control diet (D02041001B; Research Diets, New Brunswick, NJ, USA) for 3 weeks. Upon vaginal plug detection female mice were given unadulterated water or water containing $5 \mathrm{mg} / \mathrm{ml}$ metformin (Sigma-Aldrich). Water was changed weekly until mice were killed. Blood glucose levels were measured using an AlphaTRAK blood glucose meter (Abbott Laboratories, Abbott Park, IL, USA).

Metformin quantification in mouse plasma Metformin was quantified using HPLC with UV detection. Three hundred microlitres of calibrators, controls and samples were mixed with $30 \mu \mathrm{l}$ of $10 \mu \mathrm{g} / \mathrm{ml}$ phenformin (internal standard) and $1.0 \mathrm{ml}$ methanol. Samples were vortexed and centrifuged at $3200 \mathrm{~g}$ for $10 \mathrm{~min}$. Supernatant fractions were dried to residue in glass tubes, re-dissolved in $200 \mu \mathrm{l}$ of mobile phase $(35 \%$ acetonitrile, $65 \% 40 \mathrm{mmol} / 1 \mathrm{KH}_{2} \mathrm{PO}_{4}, \mathrm{pH} 4.0$ ) and filtered using a microfilterfuge tube. A $100 \mu \mathrm{l}$ volume was injected into the HPLC-UV system at room temperature with a flow rate of $1.0 \mathrm{ml} / \mathrm{min}$ and $234 \mathrm{~nm}$ wavelength of absorbance. The ratio of the peak area of metformin to the internal standard was compared against a linear regression of ratios of calibrators at concentrations of $0,62.5,125,250,1000,2000$ and $4000 \mathrm{ng} / \mathrm{ml}$. The HPLC-UV system consisted of a Dionex Omnipac PCX 500 column $(4.6 \times 250 \mathrm{~mm})$ (Thermo Fisher Scientific, Sunnyvale, CA, USA) and a Waters 2487 UV detector, 717 autosampler and 515 HPLC pump (Waters Corporation, Milford, MA, USA).

Morphometric analysis and immunostaining Pancreatic rudiments were dissected from C57Bl6 mouse embryos at E14.0. Embryonic rudiments were fixed in 3.7\% formalin in PBS then pre-embedded in Histogel (Thermo Scientific) for paraffin embedding. Newborn mouse pancreases, harvested on postnatal day 1 (P1), were fixed in $3.7 \%$ formalin in PBS for $6 \mathrm{~h}$ before embedding.

The entire pancreatic bud and neonatal pancreases were sectioned at $5 \mu \mathrm{m}$ thickness. For the in vitro study every other section of the bud was stained for PDX1 and KI-67 (8-16 sections counted per bud). Alternate sections were stained for NGN3 and KI-67. For the in vivo studies at E14.0, four sections were taken from each quartile of the organ. For the neonatal studies, five sections were taken at equal intervals [28]. Sections were deparaffinised, rehydrated and incubated overnight at $4^{\circ} \mathrm{C}$ with primary antibodies as previously described [29]. Specific primary antibodies used were insulin (guinea pig; Dako, Glostrup, Denmark), KI-67 (rabbit; Vector Laboratories, Burlingame, CA, USA), E-cadherin (mouse; 
BD Biosciences, San Jose, CA, USA), PDX1 (rabbit; Millipore, Temecula, CA, USA), NGN3 (mouse; Beta Cell Biology Consortium, www.betacell.org), phospho-S6 (rabbit, Ser240; Cell Signaling, Danvers, MA, USA) and these were followed by secondary antibodies conjugated to FITC, aminomethyl coumarin acetate (AMCA) or Cy3 (Jackson Immunoresearch, West Grove, PA, USA). TUNEL staining was performed using the ApopTag kit (Millipore, Billerica, MA, USA). Images were acquired using a Leica DM5500B fluorescence microscope (Leica Microsystems, Wetzlar, Germany).

For neonates, the areas of the pancreatic section and of insulin-positive tissue were assessed in five independent sections. For proliferation analysis at E14.0, KI-67-positive $\left(\mathrm{KI}-67^{+}\right)$nuclei were hand-counted from four sections. The total number of nuclei was counted using ImageJ64 software (imagej.nih.gov/ij/) [30]. In neonates ( $n=4$ or 5 per group), a total of $1000-3000$ beta cells were manually counted from three sections. The number of mesenchymal cells was calculated by subtracting the total number of $\mathrm{PDX} 1^{+}$cells from the total number of cells staining positive for DAPI $\left(\mathrm{DAPI}^{+}\right)$. For determination of cell size at E14.0 the area of each E-cadherinpositive $\left(\right.$ E-cadherin $\left.{ }^{+}\right)$cell was directly measured in 25 cells per bud from pancreatic sections using the magic wand tool in Photoshop CS4 (Adobe, San Jose, CA, USA) to assess the area of the cytoplasm delimited by the E-cadherin staining.

Immunoblotting Immunoblotting was performed as previously described [20,31]. Briefly, whole embryonic pancreases at E13.0 pooled from three dams were cultured in collagen for 3 days, then removed from collagen gel and immediately suspended in RIPA lysis buffer and sonicated. Thirty micrograms of protein lysates were used for the western blots using phospho-S6 (p-S6, Ser 240) and phosphorylated acetyl-CoA carboxylase (p-ACC) (Cell Signaling) antibodies. Mouse Cyclophilin B (Fisher Scientific, Pittsburgh, PA, USA) was used as a loading control.

Statistical analysis Statistical significance was assessed by the Mann-Whitney test ( $U$ test) or $t$ test, where appropriate, using GraphPad Prism (version 6.0c; GraphPad Software, La Jolla, CA, USA). Results were considered significant with a $p$ value $<0.05$.

\section{Results}

Metformin increases the number of pancreatic progenitors in vitro To assess the effect of metformin during embryonic development, E13.0 pancreatic rudiments were cultured with metformin ( $2 \mathrm{~mol} / \mathrm{l})$ or vehicle (DMSO) for $72 \mathrm{~h}$ (Fig. 1a). Buds exposed to metformin were strikingly larger than control a
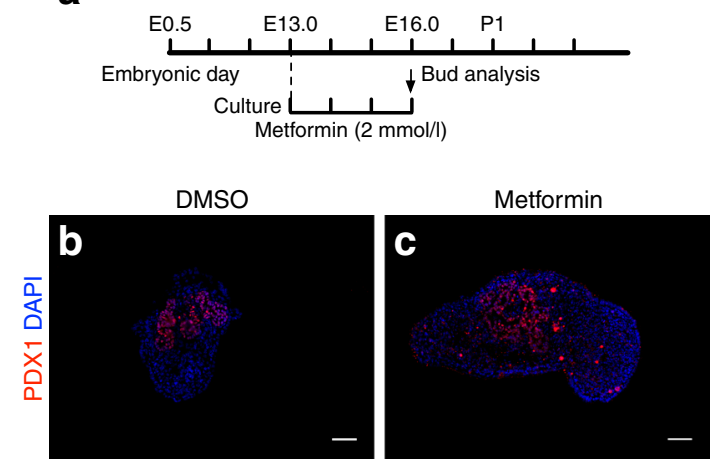

d

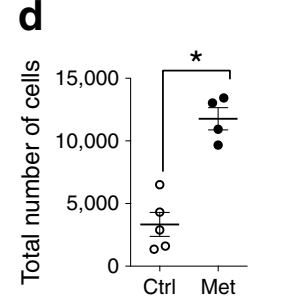

e $\mathbf{f}$

f
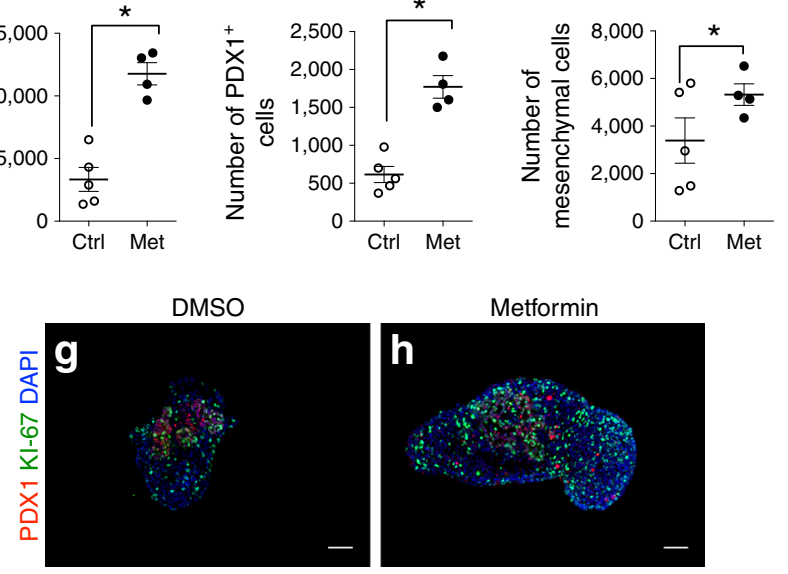

i

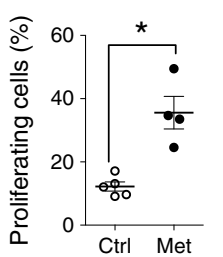

j

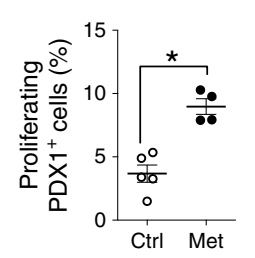

$\mathbf{k}$

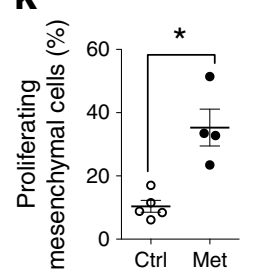

Fig. 1 Metformin increases the number and proliferation of pancreatic progenitors in vitro. (a) Schematic representation of in vitro experiments. $(\mathbf{b}, \mathbf{c})$ E13.0 buds cultured for $72 \mathrm{~h}$ with DMSO (b) or metformin (c) and stained for PDX1 (red) and a nuclear marker, DAPI (blue). (d-f) Quantification of the total cell number (d) and number of PDX $1^{+}$cells $(\mathbf{e})$ and mesenchymal cells (f). (g, h) E13.0 pancreatic buds exposed to DMSO (g) or metformin (h) and stained for PDX1 (red), KI-67 (green) and DAPI (blue). (i-k) Overall proliferation rates at the end of the culture (i), of PDX1 cells (j) and mesenchymal cells (k). ${ }^{*} p<0.05$ for the indicated comparison; scale bars, $50 \mu \mathrm{m}$. Ctrl, control (DMSO); Met, Metformin

buds (Fig. 1b, c). The total number of cells counted throughout the bud was higher in the metformin-exposed group (Fig. 1d). To determine the cause of the size difference, we examined the number of $\mathrm{PDX}^{+}$progenitors and found it to be increased in the metformin-exposed buds (Fig. 1e). Exposure to metformin also increased the number of mesenchymal cells (Fig. 1f). 
We next assessed the contribution of proliferation to the increased cell populations observed. Metformin-exposed pancreatic buds displayed an increased proliferation rate compared with controls (Fig. 1g-i). The metformin-exposed rudiments also showed an increase in $\mathrm{PDX1}^{+}$(Fig. 1j) and mesenchymal cell proliferation (Fig. 1k). These studies suggest that metformin increases the size of the pancreatic bud by increasing the number and proliferation of mesenchymal and epithelial cells.

Metformin decreases the number of endocrine progenitors in vitro We then examined the effect of metformin on the number and proliferation of the endocrine progenitor NGN3 cells. The fraction of NGN3-positive $\left(\mathrm{NGN}^{+}\right)$cells was decreased in the metformin-exposed rudiments compared with controls (Fig. 2a-c). Proliferation of the $\mathrm{NGN}^{+}$progenitors at the end of the culture, however, was unchanged (Fig. 2d-f). This suggests that the increase in proliferation of $\mathrm{PDX}^{+}$progenitors delays the differentiation to $\mathrm{NGN}^{+}$cells.

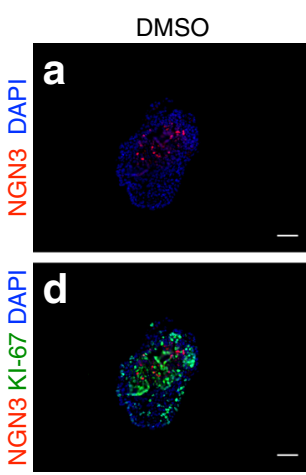

DMSO
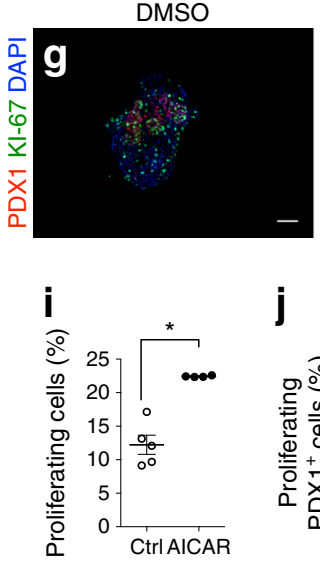

j

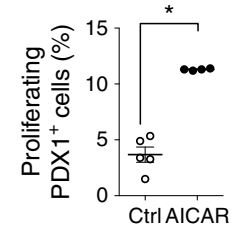

k

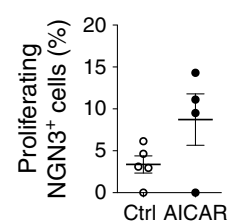

Fig. 2 Metformin decreases endocrine progenitors in vitro and AICAR increases $\mathrm{PDX}^{+}$progenitor proliferation in vitro. $(\mathbf{a}, \mathbf{b}) \mathrm{E} 13.0$ control (DMSO-treated) (a) or metformin-treated (b) buds stained for NGN3 (red) and DAPI (blue). (c) The fraction of $\mathrm{NGN}^{+}$cells. (d, e) Staining for NGN3 (red) and KI-67 (green) in the control (d) and metformin group (e). (f) The proliferation of $\mathrm{NGN3}^{+}$cells. (g, h) E13.0 control buds (g) or buds exposed to AICAR (h) stained for PDX1 (red), KI-67 (green) and DAPI (blue). (i-k) Proliferation rates at the end of the culture (i), of $\mathrm{PDX}^{+}(\mathbf{j})$ and $\mathrm{NGN}^{+}$cells $(\mathbf{k}){ }^{*} p<0.05$ for the indicated comparison; scale bars, $50 \mu \mathrm{m}$. Ctrl, control; Met, metformin
AICAR increases the bud size and rate of proliferation in vitro Metformin regulates multiple intracellular processes by inducing changes in signalling pathways including AMPK and mitochondrial function. To understand the mechanisms responsible for the effect of metformin on pancreas development, we assessed the contribution of AMPK by culturing pancreatic buds with AICAR, an AMPK activator. Buds exposed to AICAR were larger than those exposed to DMSO control (Fig. 2g, h), similar to the findings with metformin. The overall proliferation rate of bud cells exposed to AICAR was increased compared with controls (Fig. 2i). $\mathrm{PDX1}^{+}$cell proliferation was also increased, as seen with metformin (Fig. 2j). Thus, treatment of embryonic pancreas rudiments with an AMPK activator had a similar effect on bud size, $\mathrm{PDX}^{+}$cell number and proliferation to that of treatment with metformin. Also similar to the results with metformin there was no change in the proliferation rate of the $\mathrm{NGN}^{+}$cells (Fig. 2k).

Metformin exposure in utero does not alter birth variables The previous studies showed that metformin had a significant effect on the growth of the embryonic pancreas in vitro. We next sought to determine whether metformin administration in vivo recapitulated these changes. Metformin was given to pregnant dams in drinking water at a dose that had been demonstrated to yield a blood level in the human therapeutic range (equivalent to $0.03 \mathrm{mmol} / \mathrm{l}$ ) [32, 33]. Pregnant dams were exposed to metformin throughout gestation, from E0.5 (day of the vaginal plug) to delivery of the pups (Fig. 3a). The weight gain of metformin-treated dams was equal to that of the control-treated dams and the two groups displayed a similar change in blood glucose at G14.0 (Fig. 3b, c). There was no difference in litter size between the two groups (Fig. 3d). Plasma analysis revealed that metformin levels were in the upper therapeutic range in dams at delivery, and at the lower therapeutic limit in neonates (Fig. 3e).

Gestational metformin exposure increases the number of pancreatic progenitors in vivo Given the augmentation in pancreatic bud size and proliferation of pancreatic progenitors seen in vitro, we then looked for alterations in pancreatic development at E14.0. E14.0 was selected as a time when there is a high number of undifferentiated $\mathrm{PDX}^{+}$cells and when $\mathrm{NGN}^{+}$cells begin appearing in increasing numbers, just before the secondary transition for endocrine differentiation [34]. Metformin was administered to pregnant dams from E0.5 to E14.0, at which point embryonic pancreases were harvested. Upon microscopic examination of the E14.0 embryonic pancreases, metformin-exposed buds were again noted to be larger than controls (Fig. 4a, b). The total number of cells across the bud was significantly higher in the metforminexposed pancreases (Fig. 4c). Assessment of the total number of PDX $1^{+}$cells showed this to be significantly increased in the 
a

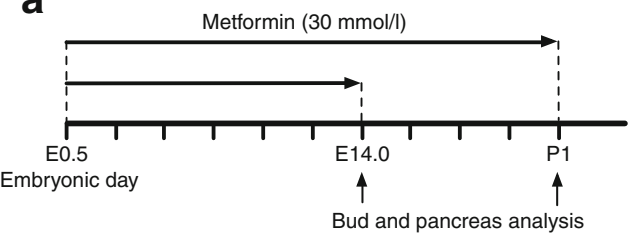

b

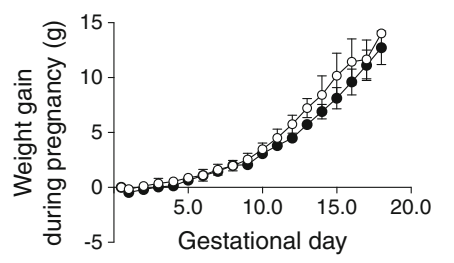

c

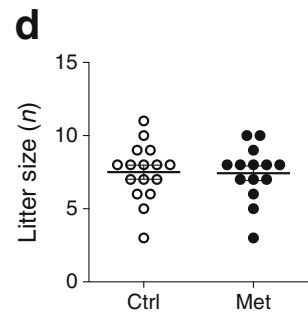

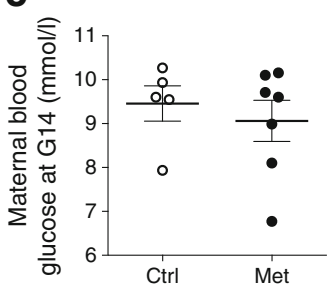

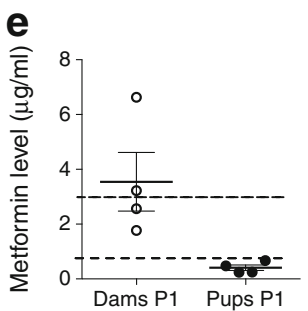

Fig. 3 Characteristics of dams and offspring after gestational exposure to metformin. (a) Schematic representation of in vivo experiments. (b) Gestational weight gain, from baseline, in control (open circles) or metformin-treated (black circles) dams $(n=5)$. (c, d) Maternal blood glucose at G14.0 (c) and litter size (d). (e) Metformin level in pregnant dams and offspring. The dashed lines indicates the therapeutic window of metformin. Ctrl, control; Met, metformin

metformin-exposed group (Fig. 4d). The total number of mesenchymal cells, however, was not altered by exposure to metformin (Fig. 4e).

The rate of proliferation was then quantified in the different compartments of the embryonic pancreas. Overall, the percentage of proliferating cells in the embryonic rudiment was the same in both conditions (Fig. $4 \mathrm{f}-\mathrm{h}$ ) and there was no significant difference in the proliferation rates of the epithelial (E-cadherin $^{+}$cells, Fig. 4i) or mesenchymal cells between the groups (Fig. 4j). We also assessed the contribution of apoptosis during development to the changes induced by metformin but found no difference in apoptosis of the $\mathrm{PDX} 1^{+}$progenitors (Fig. 4k). Thus, exposure of the developing pancreas to metformin in vivo does not alter the rate of proliferation or apoptosis when assessed at E14.0.

Gestational metformin exposure increases the number of endocrine progenitors in vivo We next examined the effect of metformin on the endocrine precursors and found that the total number of $\mathrm{NGN}^{+}$endocrine progenitors across the bud was significantly increased in the metformin-exposed group compared with the control group (Fig. $5 \mathrm{a}-\mathrm{c}$ ). $\mathrm{NGN}^{+}$cells did not
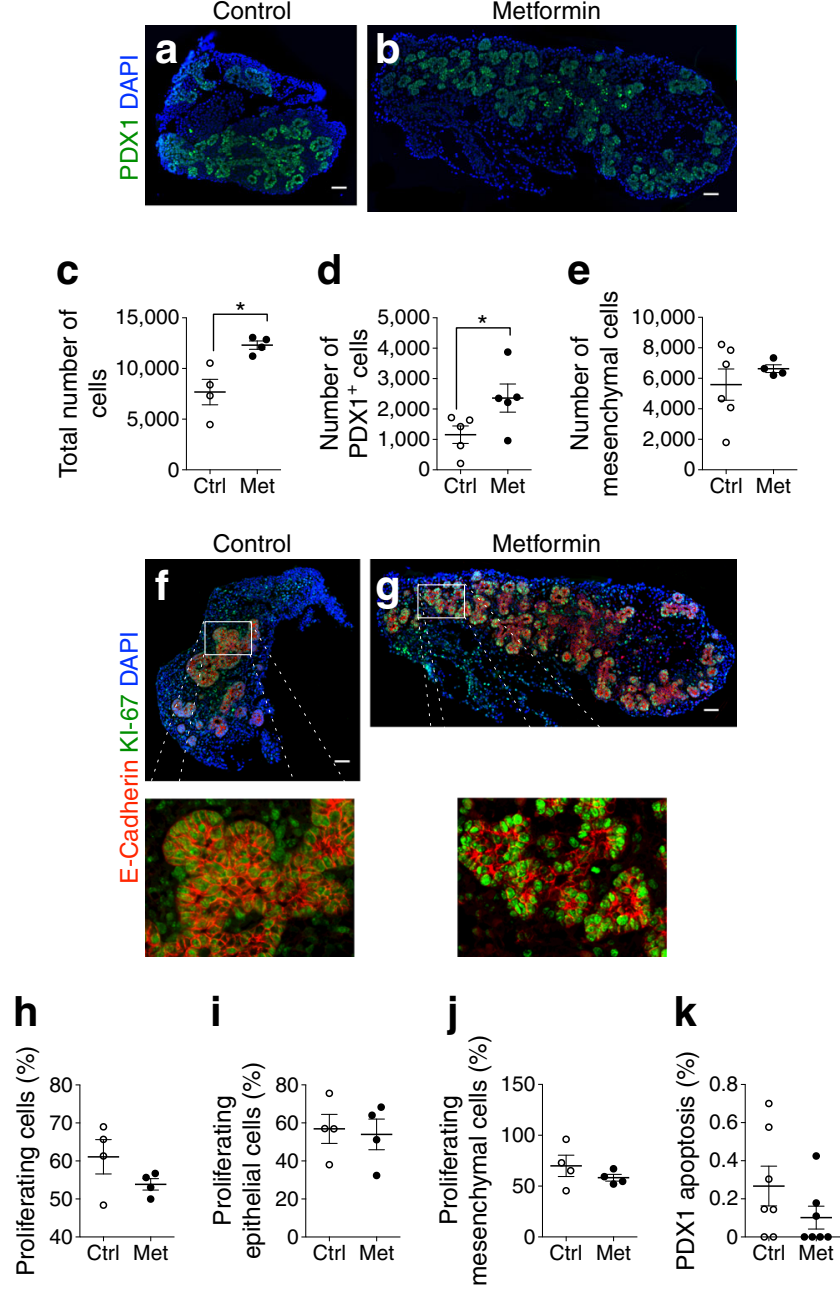

Fig. 4 Metformin increases bud size and number of pancreatic progenitors in vivo. (a, b) E14.0 embryonic pancreas, unexposed (a) or exposed to metformin (b), was stained for PDX1 (green) and DAPI (blue). (c-e) Results for: total number of bud cells (c); number of PDX $1^{+}$cells $(\mathbf{d})$ and number of mesenchymal cells (e). (f, g) E14.0 control (f) and metforminexposed buds (g) stained for E-cadherin (red), KI-67 (green) and DAPI (blue), with higher-magnification views also shown. (h-j) Proliferation rates for: total bud cells (h); epithelial cells (i) and mesenchymal cells (j). (k) Apoptosis rate for PDX $1^{+}$cells as measured by TUNEL. ${ }^{*} p<0.05$ for the indicated comparison; scale bars, $50 \mu \mathrm{m}$. Ctrl, control; Met, metformin

display a higher proliferation rate at E14.0 when the mother had received metformin (Fig. 5d-f).

Gestational metformin exposure alters intracellular signals downstream of mTORC1 To find out whether the changes observed in the developing pancreases could be due to altered mTOR signalling, we assessed the expression of phosphorylated ribosomal protein S6 (p-S6) at E14.0 on embryonic tissue sections from metformin-exposed animals. While this approach is not quantitative, pancreases from the metforminexposed embryos displayed increased staining intensity for p-S6 (Fig. 6a, b). The average cell area of the epithelial (E-cadherin ${ }^{+}$) cells was also increased (Fig. 6c-e), as would 

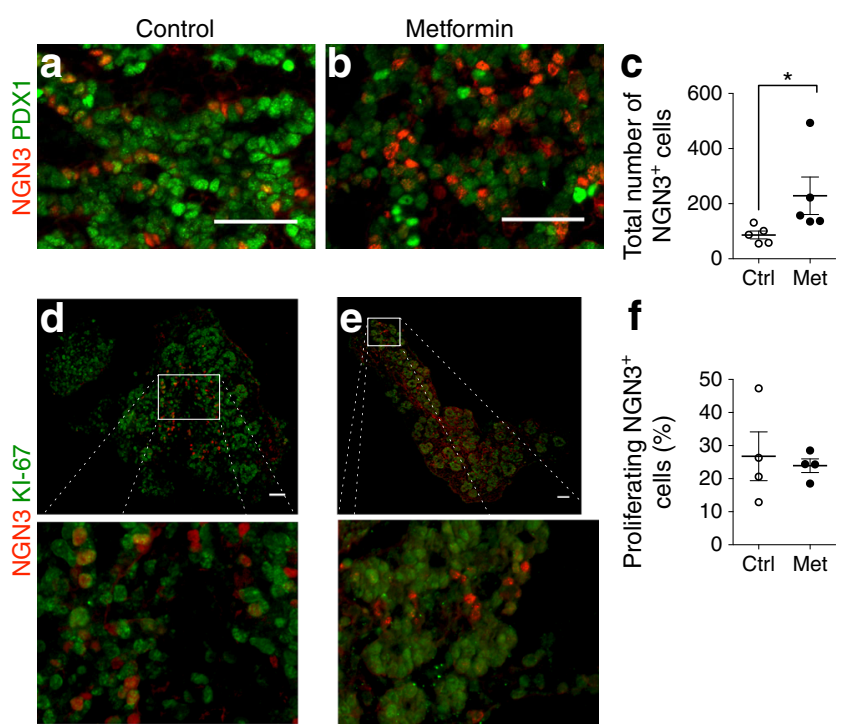

Fig. 5 Metformin increases the number of endocrine progenitors in vivo. (a, b) E14.0 control buds (a) or metformin-exposed buds (b) were stained for PDX1 (green) and NGN3 (red). (c) Total number of $\mathrm{NGN}^{+}$cells. (d, e) Staining for NGN3 (red) and KI-67 (green) in control (d) and metformin-exposed pancreases (e) at low power and high power. (f) Proliferation of $\mathrm{NGN}^{+}$cells. ${ }^{*} p<0.05$ for indicated comparison; scale bars, $50 \mu \mathrm{m}$. Ctrl, control; Met, metformin

be expected from the increased S6 activity. Finally embryonic pancreases were dissected at E13.0 and cultured with or without metformin for $72 \mathrm{~h}$. At the end of the experiments, western blots indicated that there was an increase in p-ACC (known target of metformin, used as a control) and p-S6 serine 240 in the metformin-treated rudiments (Fig. 6f).

Gestational metformin exposure leads to an increase in neonatal beta cell fraction Both in vivo and in vitro experiments indicated that the developing pancreatic rudiment grows larger under metformin treatment. The in vitro experiments also led us to hypothesise that acute metformin treatment could delay the differentiation of the endocrine progenitors while significantly increasing the number of undifferentiated $\mathrm{PDX} 1^{+}$progenitors. Given these findings, we sought to determine the extent to which these changes might result in altered beta cell fraction at birth.

Offspring of mothers characterised above were killed on the morning of birth (P1). Metformin-exposed dams delivered pups with no difference in birth length or weight compared with pups from control dams (Fig. 7a, b). The blood glucose was significantly decreased in metformin-exposed neonates (Fig. 7c).

The pancreases of offspring were analysed to establish the beta cell fraction and beta cell and acinar proliferation rates. Metformin exposure throughout gestation led to a significant increase in the beta cell fraction at birth in metformin vs control pups (Fig. $7 \mathrm{~d}-\mathrm{f}$ ) but there was no increase in the overall size of the pancreas estimated by the total cross-
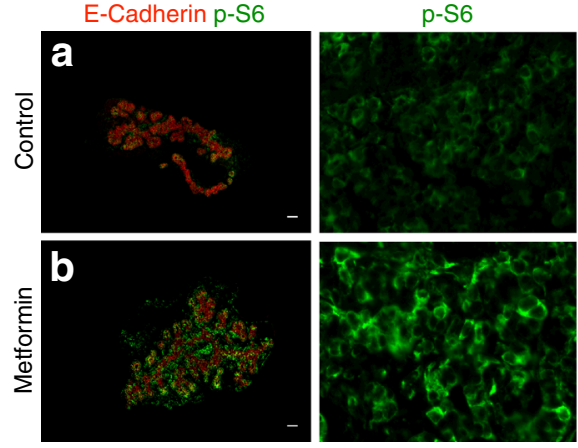

E-Cadherin p-S6
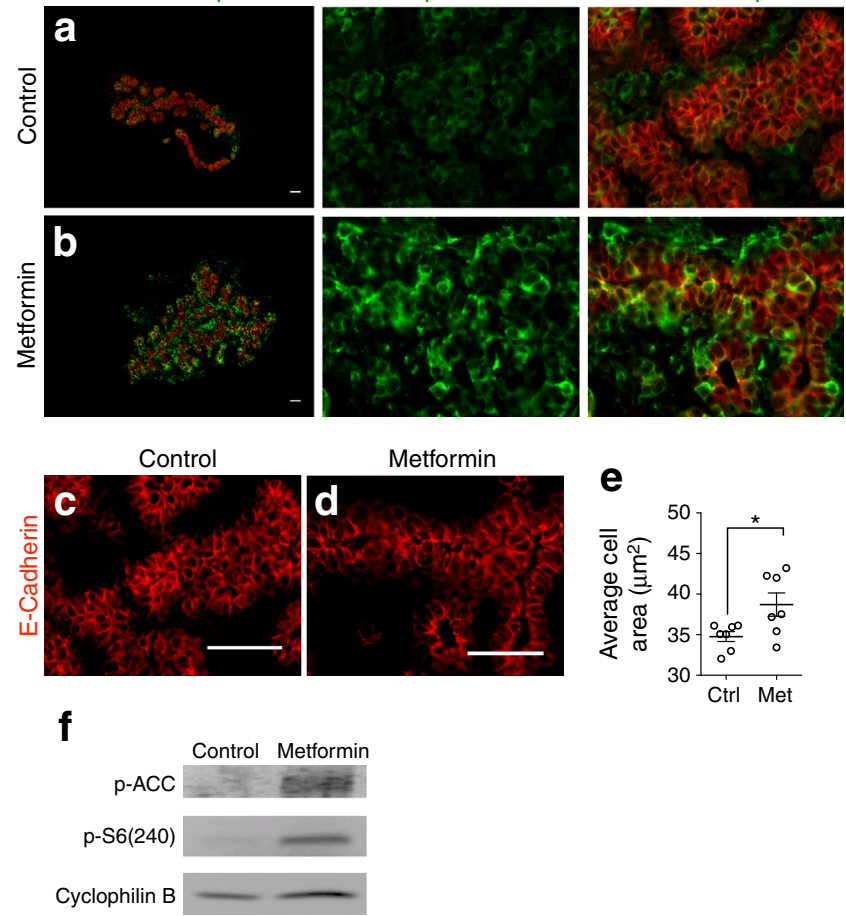

Fig. 6 Metformin exposure increases mTORC1 signalling. (a, b) E14.0 control (a) or metformin buds (b) stained for E-cadherin (red) and p-S6 (green). (c, d) High-power images from control (c) and metforminexposed buds (d) stained for E-cadherin (red). (e) Results were quantified for average cell area. (f) Representative immunoblotting for p-ACC and p-S6 from E13.0 pancreatic buds cultured with or without metformin for 72 h. * $p<0.05$ for indicated comparison; scale bars, $50 \mu \mathrm{m}$. Ctrl, control; Met, metformin

sectional area of the representative images (Fig. $7 \mathrm{~g}$ ). Assessment of beta cell and acinar proliferation rates at birth also indicated no differences between groups (Fig. 7h, i). A summary of the findings in each set of experiments is presented in Table 1.

\section{Discussion}

The studies presented here provide surprising evidence of a previously unreported effect of metformin - modulation of the developmental programme of the pancreas. The studies using an in vitro culture system demonstrate that direct treatment of embryonic pancreas with metformin enhances the proliferation and number of $\mathrm{PDX}^{+}$and mesenchymal cells. In the in vivo model, maternal exposure to metformin also induced the number of $\mathrm{PDX}^{+}$and $\mathrm{NGN3}^{+}$cells at E14.0. Continuing the treatment throughout gestation led to an increase in the beta cell fraction at birth. These findings are summarised in Table 1 and suggest that a commonly used glucose-lowering agent alters the developmental programme of the embryonic pancreas in favour of increasing beta cell 


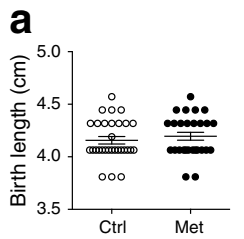

b

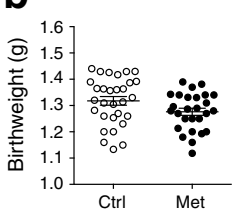

C

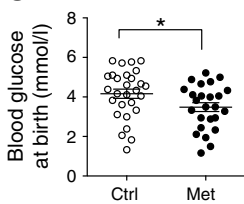

Control

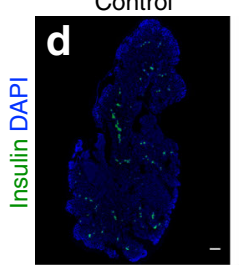

Metformin

e
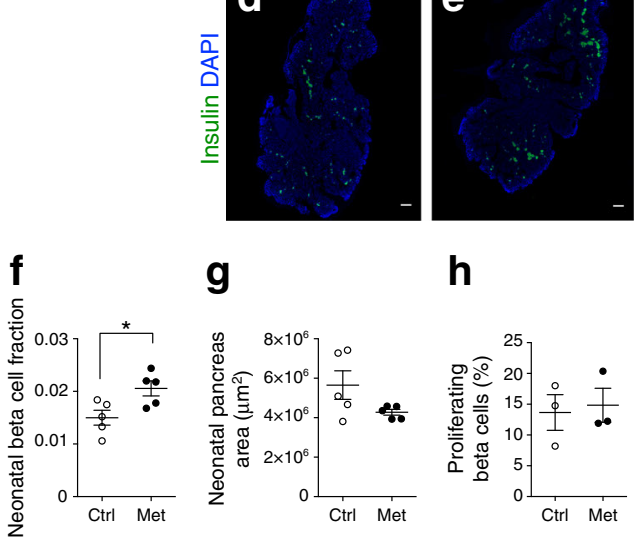

g

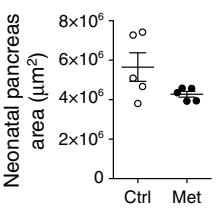

h

i

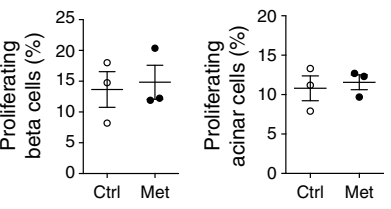

Fig. 7 Metformin exposure in utero increases beta cell fraction at birth. (a-c) Body length (a), body weight (b) and blood glucose (c) of control or metformin-treated offspring on their day of birth. (d, e) Staining of neonatal pancreases for insulin (green) and DAPI (blue) for control (d) and metformin-exposed neonates (e). (f-i) Beta cell fraction (f), neonatal pancreas area (g), beta cell proliferation (h) and acinar proliferation (i) were quantified. ${ }^{*} p<0.05$ for indicated comparison; scale bars, $50 \mu \mathrm{m}$. Ctrl, control; Met, metformin

fraction at birth, which may then confer protection from type 2 diabetes in adulthood. These observations are clinically relevant because of the ongoing studies of metformin for use in pregnancy [25] and suggest that metformin could have beneficial effects on beta cell mass in humans.

In the in vitro culture system exposure of the developing pancreatic buds to a high dose of metformin increased the pool of $\mathrm{PDX}^{+}$progenitors and augmented $\mathrm{PDX} 1^{+}$cell proliferation. The associated decrease in $\mathrm{NGN}^{+}$cells may result from a delay in differentiation following the proliferation of the $\mathrm{PDX}^{+}$progenitors, as cell cycle exit is generally linked with differentiation [35]. It is possible that a longer incubation could have had an effect on the $\mathrm{NGN}^{+}$cell number, as pancreatic development does not occur at a typical pace in culture. Interestingly, a positive effect of metformin on the number of $\mathrm{NGN}^{+}$cells was observed in vivo at E14.0. The discrepancy of these findings could reflect a longer exposure to metformin, since the developing pancreas in the in vivo model was exposed to metformin from the very early stages of pancreatic development (E9.5). Alternatively, it is possible that the differences could reflect the different concentrations of metformin used in vivo and in vitro.

In the in vivo studies, delivery of a pharmacologically relevant dose of metformin to pregnant dams resulted in an increase in the number of PDX $1^{+}$and $\mathrm{NGN}^{+}$cells in the bud. The increase in the number of PDX1 $1^{+}$cells was not accompanied by increased proliferation suggesting that metformin induces proliferation of the $\mathrm{PDX1}^{+}$progenitors prior to E14.0, as demonstrated in vitro. In addition, there was no evidence that apoptosis contributed to the metformin-induced changes in $\mathrm{PDX}^{+} 1^{+}$progenitors. The increase in the number of both $\mathrm{PDX}^{+}$and $\mathrm{NGN}^{+}$cells is likely responsible for the increased beta cell fraction in neonates. This is consistent with previous evidence demonstrating that the initial pool of $\mathrm{PDX}^{+}$and $\mathrm{NGN}^{+}$progenitors are major determinants of the final beta cell number [36, 37]. Interestingly, the proliferation of $\mathrm{PDX}^{+}$cells in vitro was in marked contrast to the lack of alteration in $\mathrm{NGN}^{+}$cell proliferation. These intriguing findings suggest that $\mathrm{PDX} 1^{+}$progenitors are more susceptible than $\mathrm{NGN}^{+}$cells to proliferative signals induced by metformin. Another possibility may be that a rise in the proliferation of $\mathrm{NGN}^{+}$cells occurs at a later time point than was examined in this study, as the peak of $\mathrm{NGN}^{+}$cell proliferation typically occurs at E14.5 [38]. Finally, it is possible that fetal administration of metformin induces longlasting consequences that could alter early postnatal beta cell remodelling and the responses of beta cells to diabetogenic conditions. This could be tested by assessing beta cell proliferation and apoptosis during the first 4 weeks of life and by exposing these mice to a high-fat diet.

The in vitro experiments demonstrated that metformin increased mesenchymal cell number and proliferation. These results are particularly interesting, as the mesenchyme has been shown to secrete growth factors and mitogenic signals

Table 1 A summary of the morphologic findings in each group of experiments

\begin{tabular}{llllll}
\hline $\begin{array}{l}\text { Stage of } \\
\text { development }\end{array}$ & $\begin{array}{l}\text { Epithelium size } \\
\left(\mathrm{PDX1}^{+} \text {cells }\right)\end{array}$ & $\begin{array}{l}\text { Epithelium proliferation } \\
(\text { E-cadherin }\end{array}$
\end{tabular}

The groups examined were embryonic pancreas in vitro at E13.0, in vivo at E14.0 and neonatal pancreas in vivo. Variables examined were epithelium size and proliferation, and endocrine progenitor number and proliferation 
that regulate proliferation and differentiation of $\mathrm{PDX}^{+}$progenitors; this may have contributed to the effects seen in $\mathrm{PDX}^{+}$cells $[36,39,40]$. Culturing the pancreatic epithelium with metformin could allow assessment of the direct role of metformin on $\mathrm{PDX}^{+}$cell proliferation in the absence of mesenchyme.

Metformin has been shown to induce AMPK activity, but the role of AMPK signalling in the developing endocrine pancreas has not been evaluated in detail [41, 42]. Adult animals with AMPK $\alpha 1$ and $\alpha 2$ double knockdown have no change in beta cell mass when compared with controls [43]. However, no evaluation of embryonic stages or neonatal beta cell fraction was done in that model. Here we demonstrate that modulation of these signalling pathways by metformin during embryogenesis has a beneficial effect on endocrine pancreatic development. How activation by metformin induces proliferation during developmental stages is not completely understood, but it is possible that these effects could be mediated by inducing the secretion of mitogenic factors by the mesenchyme. Metformin has been shown to have a negative impact mTOR signalling through several pathways and most often demonstrates an anti-proliferative effect on cells [23, 44-48]. In these experiments we present surprising evidence suggesting that metformin increases mTORC1 signalling in the embryonic pancreas. This positive effect of metformin has been demonstrated recently in hypothalamic tissue and suggests that the responses to metformin could be tissue and developmental stage specific [49]. The effect on $\mathrm{PDX}^{+}$cell proliferation induced by metformin was reproduced after treatment with AICAR in vitro, providing strong evidence that this is an AMPK-dependent effect. The ability of AICAR to induce cell proliferation has also been demonstrated previously in an embryonic cell line [50]. Whether the effects of metformin on the embryonic pancreas are AMPK- and mTOR-dependent may be clarified by further studies using genetic mouse models.

The results of these studies revealed that in vivo metformin exposure during pancreatic development leads to an increase in the pancreatic beta cell population at birth. It remains to be demonstrated that this change persists into adult life. These findings provide important clinical information because metformin may be used during pregnancy, depending on the outcome of ongoing clinical trials. The relevance of this study to the clinical use of metformin in pregnancy is currently unknown. One important difference is that administration of metformin throughout pregnancy, as in the studies described here, is not often used in pregnant women. However, a potentially relevant clinical scenario is one in which women are treated for polycystic ovary syndrome from the first trimester to delivery, as described in some studies [24, 51-55]. Future studies will be required to explore the effect of dosing and timing of metformin treatment in diabetic women. The findings described here also underscore the importance of possible programming phenomena resulting from altering cellular energy status in both the mother and the developing fetus. Further examination of these programming phenomena is warranted and this may provide a strategy aimed at enhancing beta cell mass at birth in populations at risk for type 2 diabetes later in life.

Acknowledgements We would like to thank M. Javors (University of Texas Health Science Center, San Antonio, TX, USA) for measuring metformin levels and A. Chen (formerly of the University of Michigan, Ann Arbor, MI, USA) for sectioning the tissues used in this study. The processing and embedding of embryonic pancreas was performed in the Microscopy and Image-analysis Laboratory (MIL) at the University of Michigan, Department of Cell and Developmental Biology with the assistance of J. Poore. The MIL is a multi-user imaging facility supported by NIH-NCI 5P30CA046592-26, O'Brien Renal Center, UM Medical School, Endowment for the Basic Sciences (EBS), the CDB Department, and the University of Michigan. The neonatal tissue processing and embedding was performed at the University of Michigan Comprehensive Cancer Center Tissue Core supported by NIH-NCI 5P30CA046592-26. The authors acknowledge support from the Morphology and Image Analysis Core and Phenotyping Core from the Michigan Diabetes Research Center (MDRC) (P30 DK020572).

Funding This work was funded by National Institutes of Health Grant RO1-DK-084236 and 2R01DK073716 (to EB-M) and a supplement to this award RO1-DK-084236-03S1 supported the work of BG. BG was also supported by K12-HD028820. EUA was supported by an NIH training grant (2T32DK071212-06) and a post-doctoral fellowship from the Hartwell Foundation.

Duality of interest The authors declare that there is no duality of interest associated with this manuscript.

Contribution statement BG designed experiments, generated and analysed data and wrote and approved the final manuscript. LE and $\mathrm{CC}-\mathrm{M}$ designed experiments, generated and analysed data, assisted with manuscript preparation and approved the final version. EA helped with experimental design and manuscript preparation and approved the final version. MS, MB-R and DG performed experiments, edited the manuscript and approved the final version. EB-M conceived the study, designed the experiments, helped to prepare the manuscript and approved the final version. EB-M is the guarantor of this work.

\section{References}

1. Prentki M, Nolan CJ (2006) Islet beta cell failure in type 2 diabetes. J Clin Invest 116:1802-1812

2. Reusens B, Ozanne SE, Remacle C (2007) Fetal determinants of type 2 diabetes. Curr Drug Targets 8:935-941

3. Berney DM, Desai M, Palmer DJ et al (1997) The effects of maternal protein deprivation on the fetal rat pancreas: major structural changes and their recuperation. J Pathol 183:109-115

4. Garofano A, Czernichow P, Breant B (1997) In utero undernutrition impairs rat beta-cell development. Diabetologia 40:1231-1234

5. Petrik J, Reusens B, Arany E et al (1999) A low protein diet alters the balance of islet cell replication and apoptosis in the fetal and neonatal rat and is associated with a reduced pancreatic expression of insulinlike growth factor-II. Endocrinology 140:4861-4873 
6. Snoeck A, Remacle C, Reusens B, Hoet JJ (1990) Effect of a low protein diet during pregnancy on the fetal rat endocrine pancreas. Biol Neonate 57:107-118

7. Dahri S, Snoeck A, Reusens-Billen B, Remacle C, Hoet JJ (1991) Islet function in offspring of mothers on low-protein diet during gestation. Diabetes 40(Suppl 2):115-120

8. Dahri S, Reusens B, Remacle C, Hoet JJ (1995) Nutritional influences on pancreatic development and potential links with noninsulin-dependent diabetes. Proc Nutr Soc 54:345-356

9. Garofano A, Czernichow P, Breant B (1998) Beta-cell mass and proliferation following late fetal and early postnatal malnutrition in the rat. Diabetologia 41:1114-1120

10. Ozanne SE, Hales CN (1999) The long-term consequences of intrauterine protein malnutrition for glucose metabolism. Proc Nutr Soc 58:615-619

11. Lucas A (1991) Programming by early nutrition in man. CIBA Found Symp 156:38-50, discussion 50-35

12. Apelqvist A, Ahlgren U, Edlund H (1997) Sonic hedgehog directs specialised mesoderm differentiation in the intestine and pancreas. Curr Biol 7:801-804

13. Cano DA, Soria B, Martin F, Rojas A (2013) Transcriptional control of mammalian pancreas organogenesis. Cell Mol Life Sci 71:23832402

14. Apelqvist A, Li H, Sommer L et al (1999) Notch signalling controls pancreatic cell differentiation. Nature 400:877-881

15. Oliver-Krasinski JM, Stoffers DA (2008) On the origin of the beta cell. Genes Dev 22:1998-2021

16. Fraenkel M, Ketzinel-Gilad M, Ariav Y et al (2008) mTOR inhibition by rapamycin prevents beta-cell adaptation to hyperglycemia and exacerbates the metabolic state in type 2 diabetes. Diabetes 57:945957

17. Bussiere CT, Lakey JR, Shapiro AM, Korbutt GS (2006) The impact of the mTOR inhibitor sirolimus on the proliferation and function of pancreatic islets and ductal cells. Diabetologia 49:2341-2349

18. Mori H, Inoki K, Opland D et al (2009) Critical roles for the TSCmTOR pathway in beta-cell function. Am J Physiol Endocrinol Metab 297:E1013-E1022

19. Rachdi L, Balcazar N, Osorio-Duque F et al (2008) Disruption of Tsc2 in pancreatic beta cells induces beta cell mass expansion and improved glucose tolerance in a TORC1-dependent manner. Proc Natl Acad Sci U S A 105:9250-9255

20. Rachdi L, Aiello V, Duvillie B, Scharfmann R (2012) L-leucine alters pancreatic beta-cell differentiation and function via the mTor signaling pathway. Diabetes 61:409-417

21. Inoki K, Kim J, Guan KL (2012) AMPK and mTOR in cellular energy homeostasis and drug targets. Annu Rev Pharmacol Toxicol 52:381-400

22. Janjetovic K, Vucicevic L, Misirkic M et al (2011) Metformin reduces cisplatin-mediated apoptotic death of cancer cells through AMPK-independent activation of Akt. Eur J Pharmacol 651:41-50

23. Kalender A, Selvaraj A, Kim SY et al (2010) Metformin, independent of AMPK, inhibits mTORC1 in a rag GTPase-dependent manner. Cell Metab 11:390-401

24. Glueck CJ, Goldenberg N, Wang P, Loftspring M, Sherman A (2004) Metformin during pregnancy reduces insulin, insulin resistance, insulin secretion, weight, testosterone and development of gestational diabetes: prospective longitudinal assessment of women with polycystic ovary syndrome from preconception throughout pregnancy. Hum Reprod 19:510-521

25. Rowan JA, Hague WM, Gao W, Battin MR, Moore MP, Mi GTI (2008) Metformin versus insulin for the treatment of gestational diabetes. N Engl J Med 358:2003-2015

26. Cras-Meneur C, Elghazi L, Czernichow P, Scharfmann R (2001) Epidermal growth factor increases undifferentiated pancreatic embryonic cells in vitro: a balance between proliferation and differentiation. Diabetes 50:1571-1579
27. Cras-Meneur C, Li L, Kopan R, Permutt MA (2009) Presenilins, Notch dose control the fate of pancreatic endocrine progenitors during a narrow developmental window. Genes Dev 23:2088-2101

28. Bernal-Mizrachi E, Fatrai S, Johnson JD et al (2004) Defective insulin secretion and increased susceptibility to experimental diabetes are induced by reduced Akt activity in pancreatic islet beta cells. J Clin Invest 114:928-936

29. Bernal-Mizrachi E, Wen W, Stahlhut S, Welling CM, Permutt MA (2001) Islet beta cell expression of constitutively active Akt1/PKB alpha induces striking hypertrophy, hyperplasia, and hyperinsulinemia. J Clin Invest 108:1631-1638

30. Schneider CA, Rasband WS, Eliceiri KW (2012) NIH Image to ImageJ: 25 years of image analysis. Nat Methods 9:671-675

31. Blandino-Rosano M, Alejandro EU, Sathyamurthy A et al (2012) Enhanced beta cell proliferation in mice overexpressing a constitutively active form of Akt and one allele of p21Cip. Diabetologia 55: $1380-1389$

32. (2004) Clarke's analysis of drugs and poisons, 3rd edn. Moffat AC, Osselton DM, Widdop B and Galichet LY (eds). Pharmaceutical Press, London

33. Memmott RM, Mercado JR, Maier CR, Kawabata S, Fox SD, Dennis PA (2010) Metformin prevents tobacco carcinogen-induced lung tumorigenesis. Cancer Prev Res 3:1066-1076

34. Kemp JD, Walther BT, Rutter WJ (1972) Protein synthesis during the secondary developmental transition of the embryonic rat pancreas. J Biol Chem 247:3941-3952

35. Miller JP, Yeh N, Vidal A, Koff A (2007) Interweaving the cell cycle machinery with cell differentiation. Cell Cycle 6:2932-2938

36. Attali M, Stetsyuk V, Basmaciogullari A et al (2007) Control of betacell differentiation by the pancreatic mesenchyme. Diabetes 56 : $1248-1258$

37. Stanger BZ, Tanaka AJ, Melton DA (2007) Organ size is limited by the number of embryonic progenitor cells in the pancreas but not the liver. Nature 445:886-891

38. Pictet RL, Clark WR, Williams RH, Rutter WJ (1972) An ultrastructural analysis of the developing embryonic pancreas. Dev Biol 29: 436-467

39. Gittes GK, Galante PE, Hanahan D, Rutter WJ, Debase HT (1996) Lineage-specific morphogenesis in the developing pancreas: role of mesenchymal factors. Development 122:439-447

40. Bhushan A, Itoh N, Kato S et al (2001) Fgf10 is essential for maintaining the proliferative capacity of epithelial progenitor cells during early pancreatic organogenesis. Development 128:5109-5117

41. El-Mir MY, Nogueira V, Fontaine E, Averet N, Rigoulet M, Leverve $X$ (2000) Dimethylbiguanide inhibits cell respiration via an indirect effect targeted on the respiratory chain complex I. J Biol Chem 275: 223-228

42. Zhou G, Myers R, Li Y et al (2001) Role of AMP-activated protein kinase in mechanism of metformin action. J Clin Invest 108:11671174

43. Sun G, Tarasov AI, McGinty J et al (2010) Ablation of AMPactivated protein kinase alpha1 and alpha2 from mouse pancreatic beta cells and RIP2.Cre neurons suppresses insulin release in vivo. Diabetologia 53:924-936

44. Menendez JA, Oliveras-Ferraros C, Cufi S et al (2012) Metformin is synthetically lethal with glucose withdrawal in cancer cells. Cell Cycle 11:2782-2792

45. Pierotti MA, Berrino F, Gariboldi M et al (2013) Targeting metabolism for cancer treatment and prevention: metformin, an old drug with multi-faceted effects. Oncogene 32:1475-1487

46. Larsson O, Morita M, Topisirovic I et al (2012) Distinct perturbation of the translatome by the antidiabetic drug metformin. Proc Natl Acad Sci U S A 109:8977-8982

47. Ben Sahra I, Regazzetti C, Robert G et al (2011) Metformin, independent of AMPK, induces mTOR inhibition and cell-cycle arrest through REDD1. Cancer Res 71:4366-4372 
48. Dowling RJ, Goodwin PJ, Stambolic V (2011) Understanding the benefit of metformin use in cancer treatment. BMC Med 9:33

49. Kim HJ, Park EY, Oh MJ et al (2013) Central administration of metformin into the third ventricle of C57BL/6 mice decreases meal size and number and activates hypothalamic S6 kinase. Am J Physiol Regul Integr Comp Physiol 305:R499-R505

50. Jose C, Hebert-Chatelain E, Bellance N et al (2011) AICAR inhibits cancer cell growth and triggers cell-type distinct effects on OXPHOS biogenesis, oxidative stress and Akt activation. Biochim Biophys Acta 1807:707-718

51. Vanky E, Zahlsen K, Spigset O, Carlsen SM (2005) Placental passage of metformin in women with polycystic ovary syndrome. Fertil Steril 83:1575-1578

52. Vanky E, Stridsklev S, Heimstad R et al (2010) Metformin versus placebo from first trimester to delivery in polycystic ovary syndrome: a randomized, controlled multicenter study. J Clin Endocrinol Metab 95:E448-E455

53. Jakubowicz DJ, Iuorno MJ, Jakubowicz S, Roberts KA, Nestler JE (2002) Effects of metformin on early pregnancy loss in the polycystic ovary syndrome. J Clin Endocrinol Metab 87:524529

54. Khattab S, Mohsen IA, Foutouh IA, Ramadan A, Moaz M, Al-Inany H (2006) Metformin reduces abortion in pregnant women with polycystic ovary syndrome. Gynecol Endocrinol Off J Int Soc Gynecol Endocrinol 22:680-684

55. De Leo V, Musacchio MC, Piomboni P, Di Sabatino A, Morgante G (2011) The administration of metformin during pregnancy reduces polycystic ovary syndrome related gestational complications. Eur J Obstet Gynecol Reprod Biol 157: $63-66$ 